See discussions, stats, and author profiles for this publication at: https://www.researchgate.net/publication/351182322

\title{
A Note on Fractal Interpolation vs Fractal Regression
}

Article · April 2021

DOI: 10.20935/AL808

CITATIONS

READS

0

19

1 author:

Cristina Serpa

Instituto Politécnico de Lisboa

17 PUBLICATIONS 36 CITATIONS

SEE PROFILE 


\title{
ACADEMIA $\mid$ Letters
}

\section{A Note on Fractal Interpolation vs Fractal Regression}

\author{
Cristina Serpa
}

\begin{abstract}
ISEL - Instituto Superior de Engenharia de Lisboa and CMAFcIO - Centro de Matemática, Aplicações Fundamentais e Investigação Operacional
\end{abstract}

\section{Framework}

Fractals fascinates both academics and art lovers. They are a form of chaos. A key feature that distinguishes a fractal from other chaotic phenomena is the self-similarity. This is a property that consists of replicating a shape to smaller pieces of the whole. In other words, making zoom in or zoom out gives similar perspectives of the same fractal thing. We may find these shapes everywhere and nature presents many examples of fractal creations. An amazing case is the romanesque cabbage. Mandelbrot is the father of the term fractal and studied various examples (see [3] ).

Constructing a fractal is a simple task to do, just consider an initial configuration and a replication rule for smaller scales. This is how one gets, for example, the Sierpinski triangle, the dragon curve, or the Koch Snowflake. A simple rule creates complicated shapes with non-classical geometries.

Analytically, it is also possible to define fractals as solutions of a system of iterative functional equations. Barnsley defined such a system in [1]. This non-classical geometric concept has attracted many researchers when they are faced with the need to analyse real data with irregular characteristics. 
A widely used approach to measure the fractal irregularity of a data set is to estimate its Hausdorff dimension, in particular, by computing an approximation of the box dimension. This technique is relatively easy to implement and provides a good indicator of the fractality of something being analysed.

A step further is to obtain the fractal modelling of a phenomenon. Barnsley in [1] created the so-called Fractal Interpolation Functions, that are the solutions of his iterative equation systems. In practice, for a set of $n$ elements, the system has $n-1$ equations. Each equation fits two consecutive points and are compatible with the anterior and posterior equation. The compatibility conditions are formally defined in [4,5] and theoretically developed in [2]. These conditions ensure that the estimated interpolation fractal function matches the original points.

A novel method for fractal reconstruction is the fractal regression. This is the object of a research paper submitted to a scientific journal titled "Affine fractal least squares regression model". It consists of applying the regression method to the Barnsley system of equations, estimating its coefficients, with a certain number of fractal periods $p$ and up to a few fractal levels $\mathrm{L}$, such that the number of points of the dataset is $\mathrm{p}^{L}$. Since this is a brief note on the subject, it does not contain any formulas, analytical formulations, neither proofs. They are in the literature and the references.

\section{The comparison}

The two methods Fractal Interpolation Functions (FIF) and Fractal Regression Functions (FRF) are forms of fractalization of data, or fractal reconstruction. We summarize the differences between these approaches. We will refer to each method by its acronyms, respectively FIF (as in [1]) and FRF (as in the submitted paper). Although the main paper for FRF is not yet published, at the time of writing this note, there are some teaching materials explaining the method online. ${ }^{1}$

\section{- Applicability}

FIF applies to any dataset and a fractal function fitting exactly the data is obtained.

Each FRF simulation requires a number of points equal to $\mathrm{p}^{L}$, for a given/chosen $\mathrm{p}$ and $\mathrm{L}$. Depending on the data, the estimated function may not be a good approximation. For better results, it may be necessary to resize choosing another $\mathrm{p}$, and/or L, or make a shift (left or right). Higher values of $\mathrm{L}$ require stronger fractal characteristics of the data. This exigence

\footnotetext{
${ }^{1}$ In the page www.researchgate.net/profile/Cristina_Serpa there are more information and support materials about this program.
} 
may difficult the fitness of FRF for $\mathrm{L}>2$. For some data it is possible to obtain good fitness for a given $\mathrm{L}$ and poor fitness for bigger L. FRF does not give good fitting for pure periodic data (e.g., sinusoidal). In fact, a pure periodic function does not have the self-similarity replication in different scales, i.e., the replication rule retains the same scale. Eventually, we may come across with a periodic function, where each period is a fractal. In this case, the FRF simulation should be done inside its cyclic period.

- Ease of use

The definition of FIF directly gives the analytical fractal function, with corresponding coefficients, except for the vertical factors (called also by fractal coefficients, or contraction factors). These vertical factors are not given by the FIF method, because there are more parameters of the system than equations. Some researchers use empirical approaches to obtain the missing coefficients.

The analytical solution of FRF seems to be impossible to obtain, and a numerical approximation is a viable alternative. The software Fractal Real Finder was created and developed to provide researchers with a tool to get FRF coefficients, for any $\mathrm{p}$, and L up to 4 . The software gives estimatives for all coefficients.

- Fractal levels

FIF considers one fractal level, i.e., the method does not assess the self-similarity of the data.

FRF considers at least two fractal levels, expressing the self-similarity nature of the data. That is, it has at least one replication step to smaller scales.

- Fractal function

FIF gives fractal functions, with missing information on vertical factors. For complete definition of estimated fractal function is it needed to add estimatives to the fractal coefficients. In practice, if all fractal coefficients were zero, then the obtained function would be a piecewise linear function, linking each two consecutive points with a straight line. Increasing these parameters, in absolute terms, means introducing peaks in the function graph between every two consecutive points. Bigger coefficients will result in bigger fractal oscillations appearing in between points.

FRF obtains fractal functions, estimating all coefficients. If the estimated function has all fractal coefficients zero, or near zero, then FRF gives an approximation of a piecewise linear regression. Bigger fractal coefficients identified by FRF indicates that the fractal oscillations

Corresponding Author: Cristina Serpa, mcserpa@fc.ul.pt

Citation: Serpa, C. (2021). A Note on Fractal Interpolation vs Fractal Regression. Academia Letters, Article 808. https://doi.org/10.20935/AL808. 
are more prominent. The estimatives of fractal coefficients provide a way to upper estimate Hausdorff dimension.

The fractal functions modelled by FRF are not, in general, differentiable and there are several that are nowhere differentiable.

\section{Acknowledgments}

The author acknowledges partial support by National Funding from FCT - Fundação para a Ciência e a Tecnologia, under the project: UIDB/04561/2020. 


\section{References}

[1] Barnsley, M., Fractal functions and interpolation, Constr. Approx., 2 (1986), 303-329.

[2] Buescu, J., Serpa, C., Compatibility conditions for systems of iterative functional equations with non-trivial contact sets, Results Math., 76, 68 (2021).

[3] Mandelbrot, B., The Fractal Geometry of Nature, W. H. Freeman and Company, New York (1982).

[4] Serpa, C., Buescu, J., Explicitly defined fractal interpolation functions with variable parameters, Chaos Solitons Fractals, 75 (2015), 76-83.

[5] Serpa, C., Buescu, J., Constructive Solutions for Systems of Iterative Functional Equations, Constr. Approx., 45 (2017), 273-299.

\section{Reviews}

Konstantinos Masselos, University of the Peloponnese

"The paper discusses an interesting comparison of two Fractal Reconstruction methods namely Fractal Interpolation Functions (FIF) and Fractal Regression Functions (FRF). The paper is worth reading and useful."

Mark Borres, University of San Jose-Recoletos

"Very much worth of a read.

In my country, Philippines, I have a group of professionals who are establishing the science of fractal statistics. This article helps.

The statistical implications of this result can be of very good utilization especially that most data sets are non-normally behaving (but can be tested if they behave fractally). This can be a good opportunity for experts around the world to share their insights and results to better build what Benoit Mandelbrot has started."

Anonymous reviewer

Review

"As a short paper, it has basic information about fractals and interpolation or regression. It can be relevant for mathematic students." 